Article

\title{
Geometric Distribution-Based Readers Scheduling Optimization Algorithm Using Artificial Immune System
}

\author{
Litian Duan ${ }^{1}$, Zizhong John Wang ${ }^{1,2}$ and Fu Duan ${ }^{3, *}$ \\ 1 College of Information Engineering, Taiyuan University of Technology, Taiyuan 030024, China; \\ duanlitian0030@link.tyut.edu.cn \\ 2 Department of Mathematics and Computer Science, Virginia Wesleyan College, Norfolk, VA 23502, USA; \\ zwang@vwc.edu \\ 3 College of Computer Science and Technology, Taiyuan University of Technology, Taiyuan 030024, China \\ * Correspondence: duanfu@tyut.edu.cn; Tel.: +86-351-611-1255
}

Academic Editor: Yu Wang

Received: 31 August 2016; Accepted: 12 November 2016; Published: 16 November 2016

\begin{abstract}
In the multiple-reader environment (MRE) of radio frequency identification (RFID) system, multiple readers are often scheduled to interrogate the randomized tags via operating at different time slots or frequency channels to decrease the signal interferences. Based on this, a Geometric Distribution-based Multiple-reader Scheduling Optimization Algorithm using Artificial Immune System (GD-MRSOA-AIS) is proposed to fairly and optimally schedule the readers operating from the viewpoint of resource allocations. GD-MRSOA-AIS is composed of two parts, where a geometric distribution function combined with the fairness consideration is first introduced to generate the feasible scheduling schemes for reader operation. After that, artificial immune system (including immune clone, immune mutation and immune suppression) quickly optimize these feasible ones as the optimal scheduling scheme to ensure that readers are fairly operating with larger effective interrogation range and lower interferences. Compared with the state-of-the-art algorithm, the simulation results indicate that GD-MRSOA-AIS could efficiently schedules the multiple readers operating with a fairer resource allocation scheme, performing in larger effective interrogation range.
\end{abstract}

Keywords: multiple-reader environment; multiple-reader interference; geometric distribution probability function; optimization by artificial immune system

\section{Introduction}

Radio Frequency Identification (RFID) is one of the Auto Identification and Data Capture (AIDC) techniques, which enable objects to be automatically identified. With the advantages such as automation, no-contract, easy-to-implement and enough-durability, passive RFID is widely applied in various applications such as supply chain management [1], manufacturing management [2] and warehouse management [3] with objects localization [4].

According to most applications, a single reader cannot totally cover a specific area (i.e., in a warehouse, every product has a tag attached to by automatically taking stock). Correspondingly, a classical RFID scenario is usually composed of multiple readers and large quantity of passive tags: the so-called Multiple-reader Environment (MRE). In classical MRE, multiple readers are deployed in different densities with known positions to interrogate the randomized tags with fixed positions under the same RFID system. To dynamically allocate the operating resources (i.e., available time slots and frequency channels) and manage readers, centralized mechanism is usually designed to be executed by a coordinator device (i.e., polling server), which is connected to the readers through a wired 
or wireless network [5,6]. During this process, signal interference problem degrades the operating performance in efficiency and reliability, which is caused by multiple readers operating at the same time. Correspondingly, the multiple-reader interference avoidance algorithms are proposed to help coordinator device to schedule the readers operating non-simultaneously or simultaneously but at different frequency channels, which could be clustered in two categories: the control-mechanism-based ones and the scheduling-based ones.

Control-mechanism-based algorithms [7-9] allow each reader to detect the status of other readers in order to avoid the simultaneous operating. For example, Neighbor Friendly Reader Interference Avoidance Algorithm (NFRIA) utilized readers with two bistatic antennas, where one antenna is for data transmitting and the other one is for continuously receiving to detect if other readers are using the same channel when they are operating. However, NFRIA did not take full advantage of centralized mechanism and, meanwhile, it is based on a hardware assumption that makes it hard to implement in real MRE.

Scheduling-based algorithms transform the multiple-reader interference problem into an optimization problem with respect to the allocation of operating resources (e.g., [10,11]). Based on this, the number of time slots, the number of frequency channels and the position of readers are optimally allocated by using some heuristic algorithms such as genetic algorithm [12], swarm optimization algorithm [13] and artificial immune algorithms [14-17]. Among these, the artificial immune algorithms output the best performance when compared with other heuristic ones, the advantage of which is mainly embodied in outputting a larger interrogation range. In [14-17], the author introduced a reader-to-reader avoidance model formulizing from the signal-to-interference-plus-noise (SINR) view, which could perfectly describe the interference process. However, the author did not consider the non-uniformity distribution of multiple readers deployment in MRE, resulting in the unfairness of scheduling schemes since the readers with no interferences are always operating while the ones dramatically affected by interferences are almost never operating. Therefore, part of the interrogation range is actually thriftless and, meanwhile, the interfering readers are not effectively scheduled to operate as well.

In the view of scheduling-based mechanism, a Geometric Distribution-based Multiple-reader Scheduling Optimization Algorithm using Artificial Immune System (GD-MRSOA-AIS) is proposed as a resource allocation algorithm to fairly schedule the readers operating with low interferences and large effective interrogation ranges in centralized MRE. GD-MRSOA-AIS first produces several fair feasible scheduling schemes using geometric probability distribution to allocate the operating time slot and frequency channel, which could minimize the collisions among the readers contending for operating resources and therefore maximize the probability that one reader successfully gains the opportunity to operate. To optimize these feasible scheduling schemes with the objective of maximizing the total effective interrogation range, the artificial immune system optimization with immune clone, immune mutation and immune suppression is further introduced. During the mutation process in artificial immune system optimization, there are two alternative mutation operators for different cloned antibodies, where the single-bit mutation is suitable for the superior ones and the region-bits mutation is appropriate for the regular ones.

Summarizing, the contributions of this paper are as follows:

- By using signal-to-interference-plus-noise (SINR) to explain the reduction in interrogation range, a resource allocation model is constructed with two constraint conditions which are used to decrease the multiple-reader interference.

- A geometric probability function is introduced to replace the uniform probability function to produce the feasible scheduling schemes, which ensures that readers could operate in a fair way.

- Artificial immune system with clone operator, mutation operator and suppression operator is introduced to optimize the feasible scheduling schemes. In mutation process, both of the single-bit mutation operator and the region-bits mutation operator are imported to deal with the different cloned antibodies to accelerate the optimization process and convergence speed. 
The remainder of this paper is organized as follows. In Section 2, the formulized resource allocation model is constructed in the view of signal-to-interference-plus-noise (SINR) to efficiently avoid the multiple-reader interference in MRE. The structure of GD-MRSOA-AIS is introduced in details in Section 3, where a geometric distribution probability is first utilized to generate the feasible scheduling schemes and then an artificial immune system is designed to optimize these feasible scheduling schemes as optimal ones. Simulation results for GD-MRSOA-AIS are provided in Section 4 as well as a series of comparative results between the proposed one and the state-of-the-art algorithm (i.e., LIs [14]). Finally, the conclusions and future works are drawn in Section 5.

\section{Resource Allocation Model in Centralized MRE under EPCGlobal C1G2}

\subsection{General Description with Assumptions}

In MRE, each reader transmits electromagnetic wave to create the interrogation range, where the size and shape of interrogation range depends on many factors $[5,18]$ such as the antennas design (radiation pattern, gain, polarization and impedance), tag parameters (e.g., gain, matching features, and IC sensitivity), external factors (ambient conditions and noise) and readers output power suited. In most literatures [5,14-17,19], the interrogation range is defined as a perfect circumstance where the radius only depends on the reader's output power. In this paper, we also follow this assumption as UHF RFID readers with known positions and passive RFID tags with fixed positions in centralized MRE:

- Readers are randomly distributed over an area in two dimensions.

- Any reader has only two states, active and inactive, where only the readers in active operate and interfere with other active ones.

- The interference power from multiple interfering readers seen by the desired reader is additive.

\subsection{Multiple-Reader Interference Problem in Centralized MRE}

A simple centralized MRE is illustrated as Figure 1 according to the above assumptions, which is composed of one polling server, four readers in the same rated power and two target tags. Suppose that $R_{1}$ is the desired reader, $R_{1}$ could reach to the red circle as its maximum interrogation radius of $r_{\max , 1}$ without any interference. When $\mathrm{R}_{1}$ attempts to detect the target tag $\mathrm{A}$, if the signal is masked by the interfering reader $R_{2}$ and $R_{3}$ (the interfering range is represented by the black circle), the effective interrogation range $\mathrm{r}_{1}$ (blue circle) of $\mathrm{R}_{1}$ will decrease from $r_{\max , 1}$. If $r_{1}$ is smaller than the distance $x$ between $\mathrm{R}_{1}$ and tag $\mathrm{A}, \mathrm{R}_{1}$ could not detect this target tag $\mathrm{A}$. Moreover, target tag $\mathrm{B}$ located in the overlapped range of $r_{1}$ and $r_{3}$ is hardly detected due to the multiple electromagnetic waves from $\mathrm{R}_{1}$ and $R_{3}$ simultaneously operating.

The maximum interrogation radius $r_{\max , i}$ of $\mathrm{R}_{i}$ is related to the hardware design [20], which is obtained by Equation (1), where $\lambda_{i}$ is the wavelength of $R_{i}, P_{\min }$ is the minimum power required for the tag to operate, $P_{i}$ is the signal transmission power of $\mathrm{R}_{i}, M_{d}$ is the modulation depth, and $G_{T}$ and $G_{R}$ are the gains of the transmit antenna and the receive antenna, respectively.

$$
r_{\text {max }, i}=\left(\frac{\lambda_{i}}{4 \pi}\right) \sqrt{\frac{G_{T} G_{R} P_{i}}{P_{\min }} \times \frac{1-M_{d}^{4}}{\left(1+M_{d}\right)^{2}}}
$$

However, the effective interrogation radius $\mathrm{r}_{i}$ of $\mathrm{R}_{i}$ is variable as a function of signal interfering. For reader $\mathrm{R}_{i}$ interrogating the target tag at a given time slot (i.e., at the $k$ th time slot), $\mathrm{R}_{i}$ receives the backscatter signal from the tag and, meanwhile, it is also interfered by other simultaneous operating readers signal among $N_{\text {Reader }}$ readers, where a 0-1 function $\omega_{j}(k)$ (as Equation (3)) is to indicate if the reader $\mathrm{R}_{j}$ is operating at the $k$ th slot or not. To ensure that $\mathrm{R}_{i}$ could successfully detect the target tag in distance of $x$, Signal-to-Interference-Plus-Noise ratio (SINR) [21] has to exceed the minimum one of $S I N R_{\min }$ as Equation (2): 


$$
\begin{gathered}
\operatorname{SINR}_{i}\left(x, d_{j, i}\right)=\frac{B P_{i}(x)}{\sum_{j=1 \& j \neq i}^{N_{\text {Reader }}} \omega_{j}(k) I_{j}\left(d_{j, i}\right)+N o_{i}} \geq S I N R_{\text {min }} \\
\omega_{j}(k)= \begin{cases}1, & \mathrm{R}_{j} \text { operates at } k \mathrm{th} \text { slot } \\
0, & \text { Otherwise }\end{cases}
\end{gathered}
$$

where $N o_{i}$ represents the noise power of $\mathrm{R}_{i}, B P_{i}(x)$ represents backscatter signal power from the target tag as a function of the distance $x$ between the desired reader and the target tag, $I_{j}\left(d_{j, i}\right)$ represents the interference from any other $R_{j}$ as a function of the relative distance $d_{i, j}$ (as Equation (6)) between the desired reader $R_{i}$ and the other interfering reader $R_{j}$. The function [19] of $B P_{i}(x)$ and $I_{j}\left(d_{j, i}\right)$ are defined as Equations (4) and (5), respectively.

$$
\begin{gathered}
B P_{i}(x)=\alpha_{b w} E_{\text {tag }} P_{i} G_{T} G_{R}\left(P_{0} x^{-\gamma}\right)^{2} \\
I_{j}\left(d_{j, i}\right)=h P_{j} G_{T} G_{R} \beta_{\text {mask }}\left(\Delta C H_{j, i}(k)\right) P_{0} d_{j, i}^{-\gamma}, \\
\Delta C H_{j, i}(k)=\left|C H_{i}(k)-C H_{j}(k)\right| \\
d_{j, i}=\sqrt{\left(x_{i}-x_{j}\right)^{2}+\left(y_{i}-y_{j}\right)^{2}}
\end{gathered}
$$

where $\alpha_{b w}$ is the normalized spectrum power, $E_{t a g}$ is the effective power reflection coefficient of the tag, $P_{i}$ denotes the signal transmission power of $\mathrm{R}_{i}, G_{T}$ and $G_{R}$ are the gains of the transmit antenna and the receive antenna, respectively, $P_{0}$ is the referenced path loss at the distance of $1 \mathrm{~m}, \gamma$ is the path loss exponent, $x$ is the distance between $\mathrm{R}_{i}$ and the target tag, $h$ is a fading coefficient in the channel between $\mathrm{R}_{i}$ and $\mathrm{R}_{j}, \beta_{\text {mask }}(\cdot)$ is the function of a spectrum mask defined in EPCGlobal Class1 Generation 2 standard [22], $\Delta C H_{j, i}(k)$ is the frequency channel interval between $\mathrm{R}_{i}\left(\mathrm{CH}_{i}(k)\right.$ denotes the frequency channel numbers selected by $\mathrm{R}_{i}$ at the $k$ th time slot) and $\mathrm{R}_{j}\left(\mathrm{CH}_{j}(k)\right.$ denotes the frequency channel numbers selected by $\mathrm{R}_{j}$ at the $k$ th time slot) selected, and $d_{j, i}$ is the relative distance between $\mathrm{R}_{i}$ and $\mathrm{R}_{j}$.

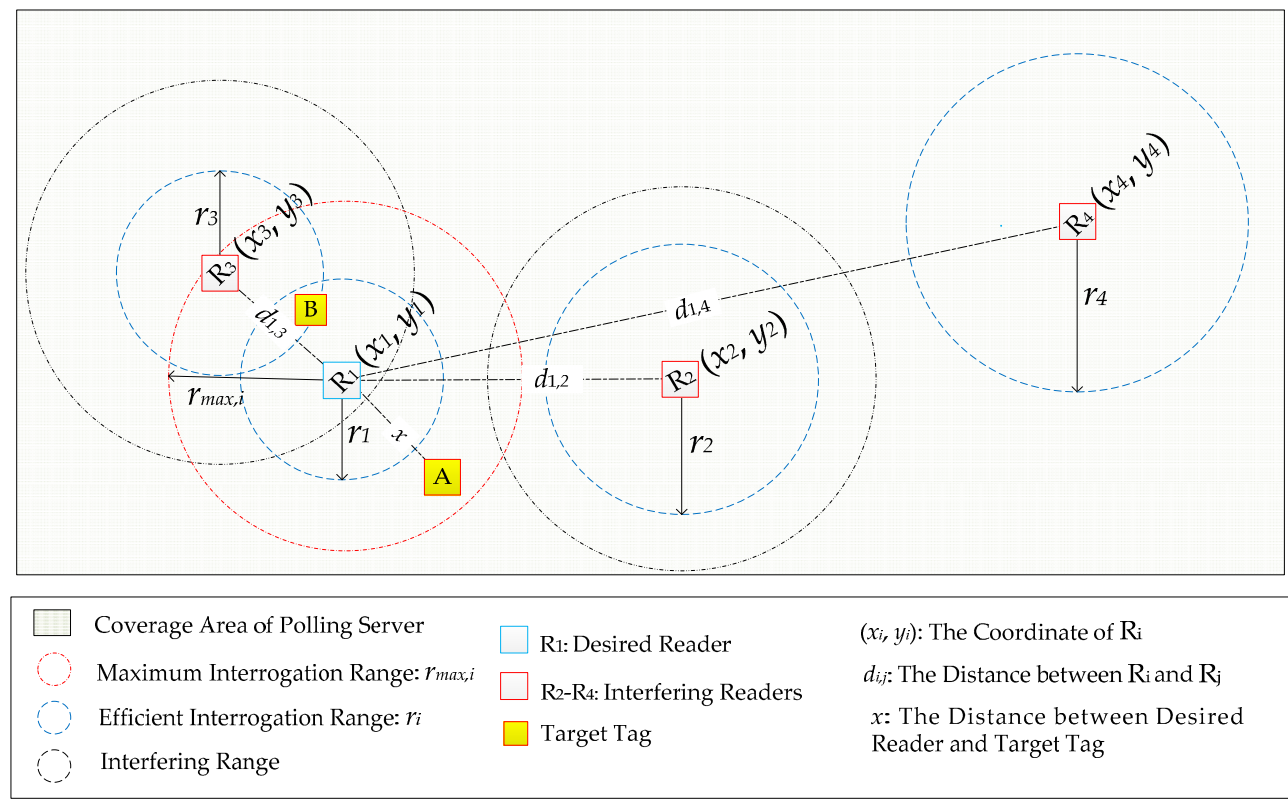

Figure 1. MRE Illustration with Centralized Mechanism.

By Equations (1)-(6), the effective interrogation range $r_{i}$ (i.e., blue circle in Figure 1 ) of $\mathrm{R}_{i}$ could be finally obtained by solving Equation (7) to ensure that the backscatter signal from target tag could be correctly recovered. 


$$
r_{i}=\min \left(\underset{x}{\operatorname{argmax}} \operatorname{SINR}\left(x, d_{j, i}\right) \geq S I N R_{\min }, r_{\max , i}\right)
$$

According to $d_{j, i}$ and $r_{i}\left(i, j \in\left[1, N_{\text {Reader }}\right]\right), \mathrm{R}_{i}$ may face two kinds of interference scenarios [23] at one time slot when it tries to detect the target tags:

- Multiple readers are simultaneously operating at the same frequency channel, which leads to the signals generated by multiple readers interfere with the reception system of the desired reader. In Figure $1, R_{1}$ suffers from this kind of interference if $R_{2}$ and $R_{3}$ are simultaneously operating with $\mathrm{R}_{1}$ at the same frequency channel. As a result, $r_{1}$ is smaller than the distance $x$ between $\mathrm{R}_{1}$ and the target tag $A, R_{1}$ could not successfully detect $A$. To ensure that $R_{1}$ could operate with a larger interrogation range to cover tag $A, R_{1}$ with $R_{2}$ and $R_{3}$ should be scheduled to operate at different frequency channels and/or at different times by polling server.

- Multiple readers operating at independently frequency channels try to simultaneously detect the same tag located in the overlapped area of their effective interrogation ranges. In Figure 1, tag B locates in the overlapped interrogation area of $R_{1}$ and $R_{3}$, where $B$ receives electromagnetic waves from both $R_{1}$ and $R_{3}$ simultaneously, it is not able to select a particular reader to communicate even if $R_{1}$ and $R_{3}$ are operating at the different frequency channels. To avoid this scenario, $R_{1}$ and $\mathrm{R}_{3}$ should operate asynchronously which are scheduled by polling server as well.

\subsection{The Proposed Resource Allocation Model in Centralized MRE}

In order to construct the resource allocation model for a centralized MRE, it is necessary to efficiently decrease the above two interference scenarios. Accordingly, Table 1 summarizes the restrictions set for any $\mathrm{R}_{i}$ and $\mathrm{R}_{j}$ operating in MRE, where $d_{f-\min }$ represents the threshold of reader-to-reader range for selecting different operating frequency channel, $d_{s-\min }\left(\Delta C H_{j, i}(k)\right)$ is the threshold of reader-to-reader range for selecting different operating time slot which depends on the selected frequency channel.

Table 1. Readers operating restrictions versus $d_{j, i}$.

\begin{tabular}{ccc}
\hline & =Frequency Channel & fFrequency Channel \\
\hline =time slot & $d_{j, i}>d_{f-\min }$ & $d_{f-\min } \geq d_{j, i}>d_{s-\min }\left(\Delta C H_{j, i}(k)\right)$ \\
$\neq$ time slot & $d_{s-\min }\left(\Delta C H_{j, i}(k)\right) \geq d_{j, i}>0$ & $d_{s-\min }\left(\Delta C H_{j, i}(k)\right) \geq d_{j, i}>0$ \\
\hline
\end{tabular}

Based on Table 1, two constraint conditions are proposed to restrict the resource allocation for readers operating as:

- $\quad \mathrm{CH}_{i}(k) \neq \mathrm{CH}_{j}(k)$ if $d_{j, i}<d_{f-\min }$

Based on the relative distance $d_{j, i}$ between $\mathrm{R}_{j}$ and $\mathrm{R}_{i}$, every $\mathrm{R}_{i}$ exists a neighbor set (denoted as $I S_{R i}=\left\{j \mid j \in\left[1, N_{\text {Reader }}\right], d_{j, i}<d_{f-m i n}\right\}$ with cardinality $\left.\left|I S_{R i}\right|=N_{\text {Neighbor }}(i), i \in\left[1, N_{\text {Reader }}\right]\right)$ to mark the readers located in the range of $d_{f-\text { min }}$, where $\mathrm{R}_{i}$ is suffered from the first kind interference if the readers from $I S_{R i}$ are simultaneously operating at the same frequency channel with $\mathrm{R}_{i}$ (i.e., $\mathrm{R}_{1}$ and $\mathrm{R}_{2}$ in Figure 1). According to LIs [14], $d_{f-\min }$ is set as $16.79 \mathrm{~m}$ (the transmitting signal power of reader is $1 \mathrm{~W}$, and the frequency is $902-928 \mathrm{MHz})$ for any two $\mathrm{R}_{i}$ and $\mathrm{R}_{j}$. Therefore, $\mathrm{R}_{i}$ with any $\mathrm{R}_{j}$ in its $I S_{R i}(j \neq i)$ should be operating at different frequency channels as: $\mathrm{CH}_{i}(k) \neq \mathrm{CH}_{j}(k)$ if $j \in I S_{R i}$ and $j \neq i$, where $\mathrm{CH}_{i}(k)$ denotes the frequency channel numbers selected by $\mathrm{R}_{i}$ at the $k$ th time slot.

- $\quad \omega_{j}(k)=0$ if $d_{j, i} \leq d_{s-\min }\left(\Delta C H_{j, i}(k)\right)$

Further, in $d_{f-\min }$, another smaller distance denoted as $d_{s-\min }$ exists, where $\mathrm{R}_{i}$ is suffered from the second kind interference if readers located in $d_{s-\min }$ are operating simultaneously even if they are at different frequency channels because they are overlapped in the effective interrogation range (i.e., $R_{1}$ 
and $\mathrm{R}_{3}$ in Figure 1 ). Usually, $d_{s-\min }$ for $\mathrm{R}_{i}$ and $\mathrm{R}_{j}$ is variable along with the correspondingly selected frequency channels interval $\Delta C H_{j, i}(k)$, which is listed in Table 2. The calculation process is attached in Appendix A.

Table 2. Influence of frequency channel interval to $d_{s-m i n}$.

\begin{tabular}{cccccc}
\hline$\Delta C \boldsymbol{H}_{j, i}(\boldsymbol{k})$ & $\mathbf{0}$ & $\mathbf{1}$ & $\mathbf{2}$ & $\mathbf{3}$ & $\geq 4$ \\
\hline $\boldsymbol{d}_{s-\min }\left(\boldsymbol{\Delta C} \boldsymbol{H}_{j, i}(k)\right)$ & $0.0269 \mathrm{~m}$ & $0.8452 \mathrm{~m}$ & $2.6970 \mathrm{~m}$ & $6.7458 \mathrm{~m}$ & $10.339 \mathrm{~m}$ \\
\hline
\end{tabular}

Therefore, $\mathrm{R}_{i}$ with $\mathrm{R}_{j}$ in $d_{s-\min }$ should be operating non-simultaneously as: $\omega_{j}(k)=0$ if $d_{j, i} \leq d_{s-\min }\left(\Delta C H_{j, i}(k)\right)$, where $\omega_{j}(k)$ denotes the $0-1$ function of Equation (3).

Constricted by the constraint conditions, the resource allocation model is finally constructed as Equation (8) with the objective of maximizing the total effective interrogation range (denoted as $S$ ) to schedule $N_{\text {Reader }}$ readers operating with $N_{\text {Slot }}$ time slots and $N_{\text {Freq }}$ frequency channels. Note that $N R=\left\{1,2,3, \ldots, N_{\text {Reader }}\right\}, N S=\left\{1,2,3, \ldots, N_{\text {Slot }}\right\}, C H=\left\{1,2,3, \ldots, N_{\text {Freq }}\right\}, W S=\left\{\left(x_{i}, y_{i}\right) \mid x_{i} \in\left[0, L_{x}\right]\right.$, $\left.y_{i} \in\left[0, L_{y}\right]\right\}$ and $I S_{R i}=\left\{j \mid j \in N R, d_{j, i}<d_{f-\min }\right\}$.

$$
\left\{\begin{array}{l}
\text { Maximize } S=\sum_{i=1}^{N_{\text {Reader }}} \sum_{k=1}^{N_{\text {Slot }}} \omega_{i}(k) \pi\left(r_{i}(k)\right)^{2}, \\
\text { s.t. } \forall i, j \in N R, \quad \forall k \in N S, C H_{i}(k), C H_{j}(k) \in C H,\left(x_{i}, y_{j}\right) \in W S, \Delta C H_{j, i}(k) \neq 0 \quad \text { if } j \in I S_{R_{i}}, \\
\omega_{j}(k)=0 \quad \text { if } \quad d_{j, i} \leq d_{s-\min }\left(\Delta C H_{j, i}(k)\right)
\end{array}\right.
$$

\section{Geometric Distribution-Based Multiple-Reader Scheduling Optimization Algorithm Using Artificial Immune System (GD-MRSOA-AIS)}

According to the proposed resource allocation model, GD-MRSOA-AIS is proposed to fairly allocate the operating resources with lower reader interference and larger effective interrogation range. In GD-MRSOA-AIS, it is composed of two parts:

- GD: Geometric distribution function (GD) combined with the fairness consideration is introduced to randomize the feasible scheduling schemes with lower interferences which satisfy the constraint conditions in Equation (8). Details are described in Section 3.1;

- AIS: Artificial immune system optimization (AIS) is to optimize these feasible scheduling schemes as the optimal scheduling scheme for readers fairly operating with larger effective interrogation range. Details are presented in Section 3.2.

\subsection{Feasible Scheduling by Geometric Distribution Function}

According to the constraint conditions in Equation (8), GD-MRSOA-AIS will first produce several feasible solutions as the feasible scheduling schemes to ensure that readers could fairly operate with low signal interference.

In centralized MRE, both the interference-reader (i.e., $R_{1}, R_{2}$ and $R_{3}$ in Figure 1 ) and the isolation-reader (i.e., $R_{4}$ in Figure 1 ) exist, where the isolated readers could operate at any time while the interfering readers have to be scheduled by polling server to decrease the interferences as much as possible. On the other hand, it is obvious that the isolated readers keeping on operating is thriftless since the number of deployed tags in the interrogation range is constant. Therefore, it is unfair to allocate the interfering readers and isolated readers with same opportunities to operate. To enhance the fairness, the interfering readers should gain more opportunities to operate.

To efficiently and fairly allocate the available operating time slots, a geometric probability distribution function called Sift $[24,25]$ is imported to replace the typical uniform distribution. Based on the character of Sift distribution, it could increase the probability that one contender selects one resource in a low position, winning the contention quickly. Therefore, if $\mathrm{R}_{i}$ contends for the operating time slot with large quantity of neighbor readers simultaneously, most neighbors will tend to select the last few 
time slots, and only the minority will contend for the first few time slots, decreasing the probability of collision in slots selection and increasing the probability that $R_{i}$ successfully selects one of the first few time slots. On the contrary, if $R_{j}$ contends for the operating time slots with small quantity of neighbor readers, the probability of selecting one slot almost obeys uniform distribution.

For Sift_Function, the probability that the $i$ th reader gains the opportunity to operate at the $k$ th time slot from $N_{\text {Slot }}$ time slots is denoted as Equation (9). Here, $N_{\text {Neighbor }}(i)$ is the cardinality of $I S_{R i}$ for $\mathrm{R}_{i}$, where $N_{\text {Neighbor }}(i)=1$ denotes that there is no neighbors located around $\mathrm{R}_{i}$ and therefore $\mathrm{R}_{i}$ is an isolated reader without any possible interference.

$$
p(k)_{i}=\frac{\left(1-\alpha_{i}\right) \alpha_{i}^{N_{\text {Slot }}}}{1-\alpha_{i}^{N_{\text {Slot }}}} \alpha_{i}^{-k}, 1 \leq k \leq N_{\text {Slot }}, \quad 0<\alpha_{i}<1, \alpha_{i}=N_{\text {Neighbor }}(i)^{-1 /\left(N_{\text {Slot }}-1\right)}
$$

Note that when $N_{\text {Neighbor }}(i)=1$, then $\alpha_{i}=1$, and $\lim _{\alpha \rightarrow 1} p(k)_{i}=1 / N_{\text {Slot }}$, becoming the uniform probability distribution. Consequently, $\mathrm{R}_{i}$ with bigger $N_{\text {Neighbor }}(i)$ could easily wins the $k$ th time slot as a higher $p(k)_{i}$ while $\mathrm{R}_{j}$ with smaller $N_{\text {Neighbor }}(j)$ has to select the operating time slot like uniform distribution as a lower $p(k)_{j}$.

By using Sift_Function, it generates several feasible scheduling schemes for readers operating with the allocated time slots and frequency channels, where the feasible scheduling schemes are restricted by the constraint conditions in Equation (8). Therefore, each feasible scheduling scheme produced by Sift_Function is actually a feasible solution for Equation (8), where the pseudocode is summarized as Algorithm 1.

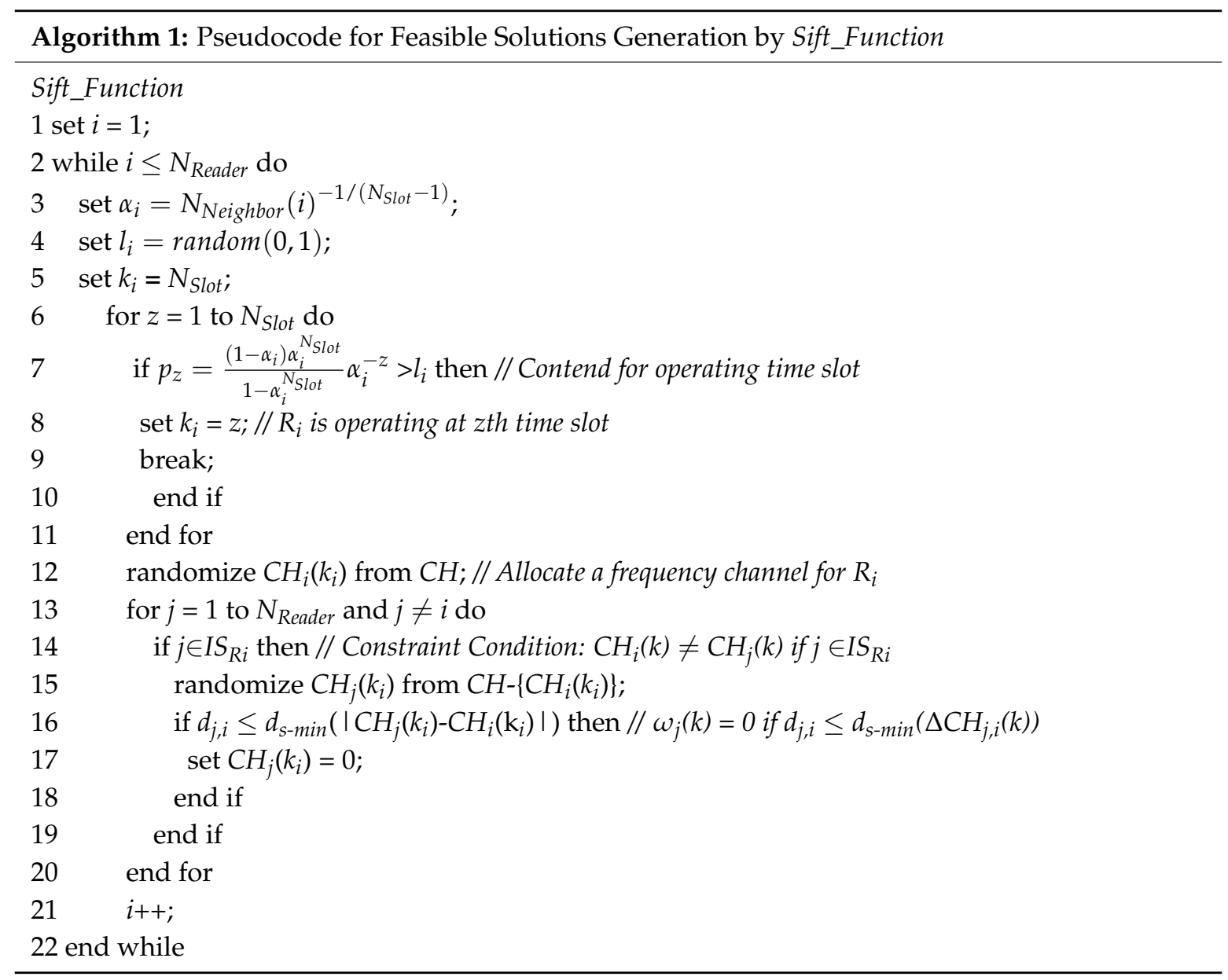


For each feasible solution of Equation (8), the total effective interrogation range might be not large enough. Therefore, this feasible scheduling scheme might be not the optimal one to maximize the global effective interrogation range. To optimize these feasible scheduling schemes, the artificial immune system optimization is introduced in the following section.

\subsection{Optimal Scheduling by Artificial Immune System Optimization}

For the produced feasible scheduling schemes, they could be optimized by using artificial immune system optimization, where each candidate antibody corresponds to one feasible solution of Equation (8) produced by Sift_Function and the antigen corresponds to the objective function of Equation (8) with the constraint conditions. According to this, the affinity function could be formulated as Equation (10), where the process for optimizing the feasible solutions of Equation (8) is transformed to find the maximum affinity value of Equation (10) and its corresponding optimum antibody.

$$
\left\{\begin{aligned}
& \text { Affinity }(A b)= \sum_{i=1}^{N_{\text {Reader }}} \sum_{k=1}^{N_{\text {Slot }}} \omega_{i}(k) \times\left(r_{i}(k)\right)^{2}, \\
& \text { s.t. } \forall i, j \in N R, \quad \forall k \in N S, \quad C H_{i}(k), C H_{j}(k) \in C H,\left(x_{i}, y_{j}\right) \in W S, \Delta C H_{j, i}(k) \neq 0 \quad \text { if } j \in I S_{R_{i}}, \\
& \omega_{j}(k)=0 \quad \text { if } d_{j, i} \leq d_{S-\min } \quad\left(\Delta C H_{j, i}(k)\right)
\end{aligned}\right.
$$

The proposed artificial immune system optimization is predefined to iterate $N_{G e n}$ generations, where it exists three phases in every iterative generation as: immune clone, immune mutation and immune suppression/recruitment. In the clone phase, each antibody is acted as a parent to be correspondingly cloned for a fixed number of $N_{\text {Clone }}$ children antibodies, and all children antibodies go through the mutation process but only one child antibody with the highest affinity-value among the children antibodies is selected to compare with its parent antibody. If this selected child antibody is superior to its parent antibody in affinity value, it will replace its parent antibody as a new generation. Once the affinity value of this new generation is not significantly different from that of the previous generation, the suppression will be triggered, where the $P_{S}$ antibodies with lowest affinity-values are suppressed. Then, the corresponding numbers of antibodies produced by Sift_Function are recruited. This process is repeated until $N_{G e n}$ iteration is met. The related symbols and their descriptions are listed in Table 3.

Table 3. Symbol and description in artificial immune system optimization.

\begin{tabular}{cc}
\hline Symbol & Description \\
\hline$A B$ & The candidate antibody population \\
$a b(u)$ & The $u$ th antibody \\
$A B_{\text {Remain }}$ & The $i$ th antibody segment in $A b(u)$ \\
$A b(u)_{\text {Clone }}$ & The set for remained antibodies \\
$A f f i n i t y(\cdot)$ & The clone pool for $A b(u)$ \\
$A f f \_$old & Affinity function \\
$A f f$ new & The mean affinity value of the previous generation \\
$C H_{i}(k)$ & The mean affinity value of the current generation \\
$N_{\text {Ini }}$ & The $k$ th bit in $a b(u)_{i}$ \\
$N_{\text {Clone }}$ & The population size of $A B$ \\
$N_{\text {Gen }}$ & The cloned multiplier \\
$L$ & The generation multiplier \\
$P_{S}$ & Region-bits mutation length \\
\hline
\end{tabular}

\subsubsection{Initialization}

To initialize the optimizing process by using artificial immune system, $N_{\text {Ini }}$ antibodies (feasible solutions) are randomized by repeatedly run Sift_Function in $N_{\text {Ini }}$ times to construct the candidate antibody population set as $A B=\left\{A b(u) \mid u \in\left[1, N_{\text {Ini }}\right]\right\}$. 
For each antibody $A b(u)$ in $A B$, it is encoded as integer type since both frequency channels and time slots are discrete distribution as integer type. Seen from Figure $2 \mathrm{a}, A b(u)$ consists of $N_{\text {Reader }}$ segments as $a b(u)_{1}, a b(u)_{2}, a b(u)_{3}, \ldots, a b(u)_{N R e a d e r}$, where each $a b(u)_{i}$ is actually corresponding to a reader $\mathrm{R}_{i}$ and it includes a sequence of bits as $\mathrm{CH}_{i}(1), \mathrm{CH}_{i}(2), \mathrm{CH}_{i}(3), \ldots, \mathrm{CH}_{i}\left(\mathrm{~N}_{\text {Slot }}\right)$ where each $\mathrm{CH}_{i}(k)$ is denoted as the selected frequency channel of reader $\mathrm{R}_{i}$ at the $k$ th time slot. $C H_{i}(k)=0$ means that reader $R_{i}$ does not operate at this $k$ th slot.

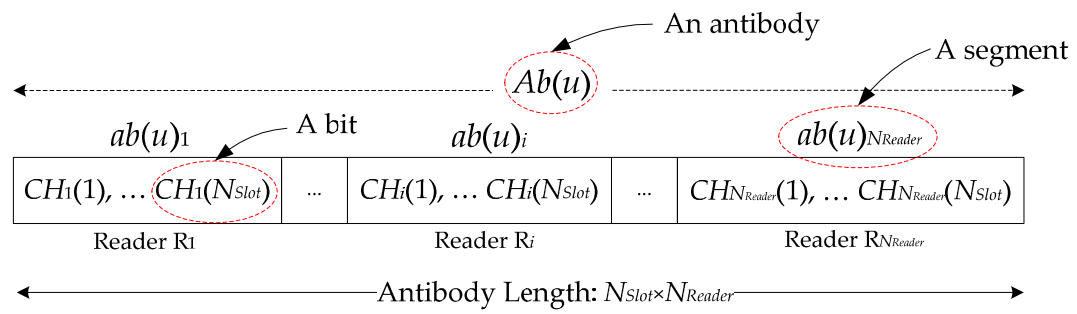

(a)

\begin{tabular}{|c|c|c|c|}
\hline$a b c(c)_{1}$ & & $a b c(c) i$ & $a b c(c)_{N_{\text {Render }}}$ \\
\hline $\mathrm{CH}_{1}(1), \ldots \mathrm{CH}_{1}(k), \ldots \mathrm{CH}\left(\mathrm{N}_{\text {slot }}\right)$ & $\ldots$ & $\mathrm{CH}_{i}(1), \ldots \mathrm{CH}_{i}(k), \ldots \mathrm{CH} \mathrm{H}_{i}\left(\mathrm{~N}_{\text {Slot }}\right)$ & 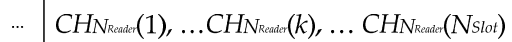 \\
\hline
\end{tabular}

(b)

Figure 2. (a) Encoding format for the $u$ th antibody individual; (b) Encoding format for the $c$ th child antibody.

\subsubsection{Clone Operation}

After producing the candidate antibody population set as $A B=\left\{A b(u) \mid u \in\left[1, N_{\text {Ini }}\right]\right\}$, each $A b(u)$ is regarded as an parent antibody. For each parent antibody $A b(u), N_{\text {Clone }}$ cloned antibodies with the same encodings are produced as its children antibodies, in which these children antibodies will go through the mutation operation to increase the diversity and therefore they have more potential to evolve as excellent individuals. Here, $N_{\text {Clone }}$ is required to be predefined as the cloned multiplier, and each $A b(u)$ has a clone pool as $A B(u)_{\text {Clone }}=\left\{A b c(c) \mid c \in\left[1, N_{\text {Clone }}\right]\right\}$ to store its children antibodies, where each $A b c(c)$ in $A B(u)_{\text {Clone }}$ is encoded as Figure $2 \mathrm{~b}$.

Note that each child antibody $A b c(c)$ from the clone pool $A B(u)_{C l o n e}$ is totally the same as its parent antibody $A b(u)$ in every bit before the mutation operation.

\subsubsection{Mutation Operation}

For each child antibody $A b c(c)$ (i.e., Figure 2b) from the clone pool $A B(u)_{\text {Clone }}=\{A b c(c) \mid c \in[1$, $\left.\left.N_{\text {Clone }}\right]\right\}$ of its parent $A b(u)$, there are two optional mutation operators as: single-bit mutation operator is applied for the ones with higher affinity values and region-bits mutation operator is applied for the ones with lower affinity values.

For single-bit mutation operator, only one bit $\mathrm{CH}_{i}(k)$ is randomly selected as a hotspot to be mutated following the constraint conditions, which belongs to a reader $\mathrm{R}_{i}$ (i.e., Figure $2 \mathrm{~b}$ ). As a result, this mutated $A b c(c)$ is also a feasible scheduling scheme as well.

For region-bits mutation operator, a contiguous region bits are selected to be mutated starting from a hotspot of $\mathrm{CH}_{i}(k)$ increasing $L$-bit length, note that $L$ is less than $N_{\text {Slot }}$ minus $k$ to guarantee the mutation is happened in one reader $\mathrm{R}_{i}$ (i.e., Figure $2 \mathrm{~b}$ ). In addition, this mutation is restricted by the constraint conditions. As a result, this mutated $A b c(c)$ is also a feasible scheduling scheme.

By single-bit mutation, the children antibody could extremely inherit the superior bits of the parent antibody while the children antibody would have more potential to mutate as the superior 
ones by region-bits mutation. Both of them could fast the convergence and quickly reach to the global optimization.

The single-bit and region-bit mutation operator run as in Algorithms 2 and 3 respectively.

After the mutation operations, for each parent antibody $A b(u)\left(u \in\left[1, N_{I n i}\right]\right)$, its children antibodies $A b c(c)$ from the clone pool $A B(u)_{\text {Clone }}=\left\{A b c(c) \mid c \in\left[1, N_{\text {Clone }}\right]\right\}$ are totally different: some $A b c(c)$ surpasses $A b(u)$ in affinity value while some of $A b c(c)$ are identical or inferior to $A b(u)$. To distinguish them, all $A b c(c)\left(c \in\left[1, N_{\text {Clone }}\right]\right)$ should be sorted by affinity value. Select the $A b c(c)$ with highest affinity value and compare it with its parent antibody $A b(u)$ : if this $A b c(c)$ is superior to its parent antibody $A b(u)$, it will replace its parent antibody as a new candidate antibody in $A B$.
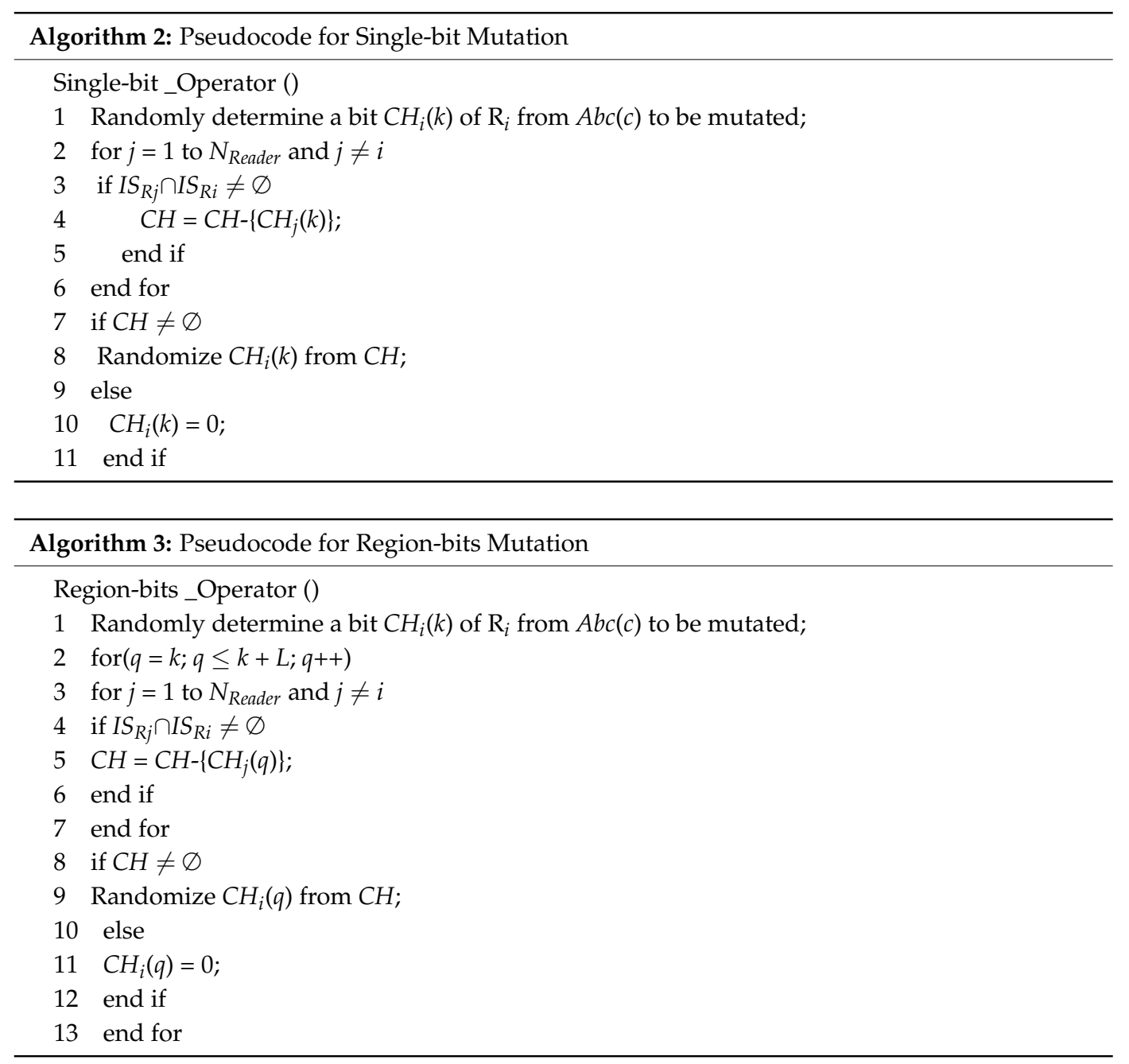

\subsubsection{Suppression Operation}

After the mutation operation, the candidate antibodies are changed. If the affinity value of new candidate antibody population set $A B$ is significantly increased, this $A B$ will be directly regarded as a new generation and go through the same immune process via clone operation, mutation operation and suppression operation.

On the contrary, the candidate antibodies with highest affinity values should be promoted while the antibodies with lowest affinity values should be suppressed. Sorted by the affinity value, it will suppress $P_{S}$ percentage of antibodies with lowest affinity values. After that, $P_{S}$ of $N_{I n i}$ antibodies (feasible solutions) are produced by Sift_Function, where these fresh candidate antibodies are recruited in $A B$ to compose a new generation population. In addition, this new generation population will go through the same immune process via clone operation, mutation operation and suppression operation. 
When the iteration reaches the predefined maximum generations $N_{\text {gen }}$, GD-MRSOA-AIS terminates. Finally, the pseudocode for proposed artificial immune system (proposed-AIS) optimization in GD-MRSOA-AIS is listed as Algorithm 4, where the corresponding flowchart is shown in Figure 3.

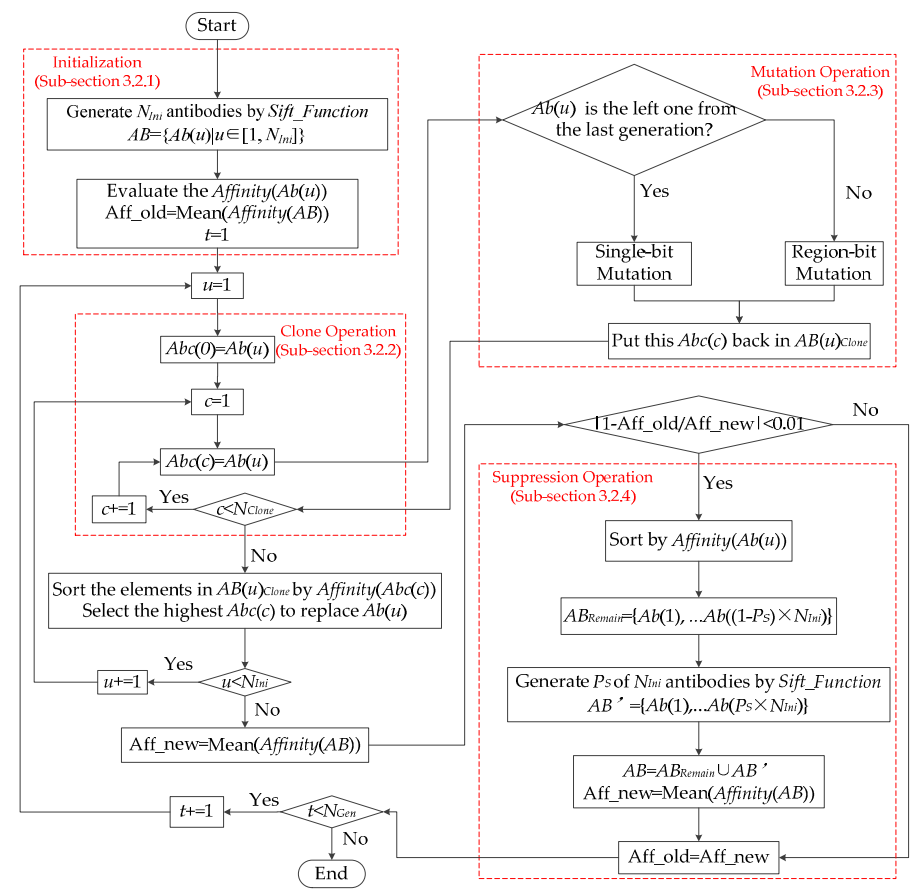

Figure 3. Flowchart of proposed AIS optimization in GD-MRSOA-AIS.

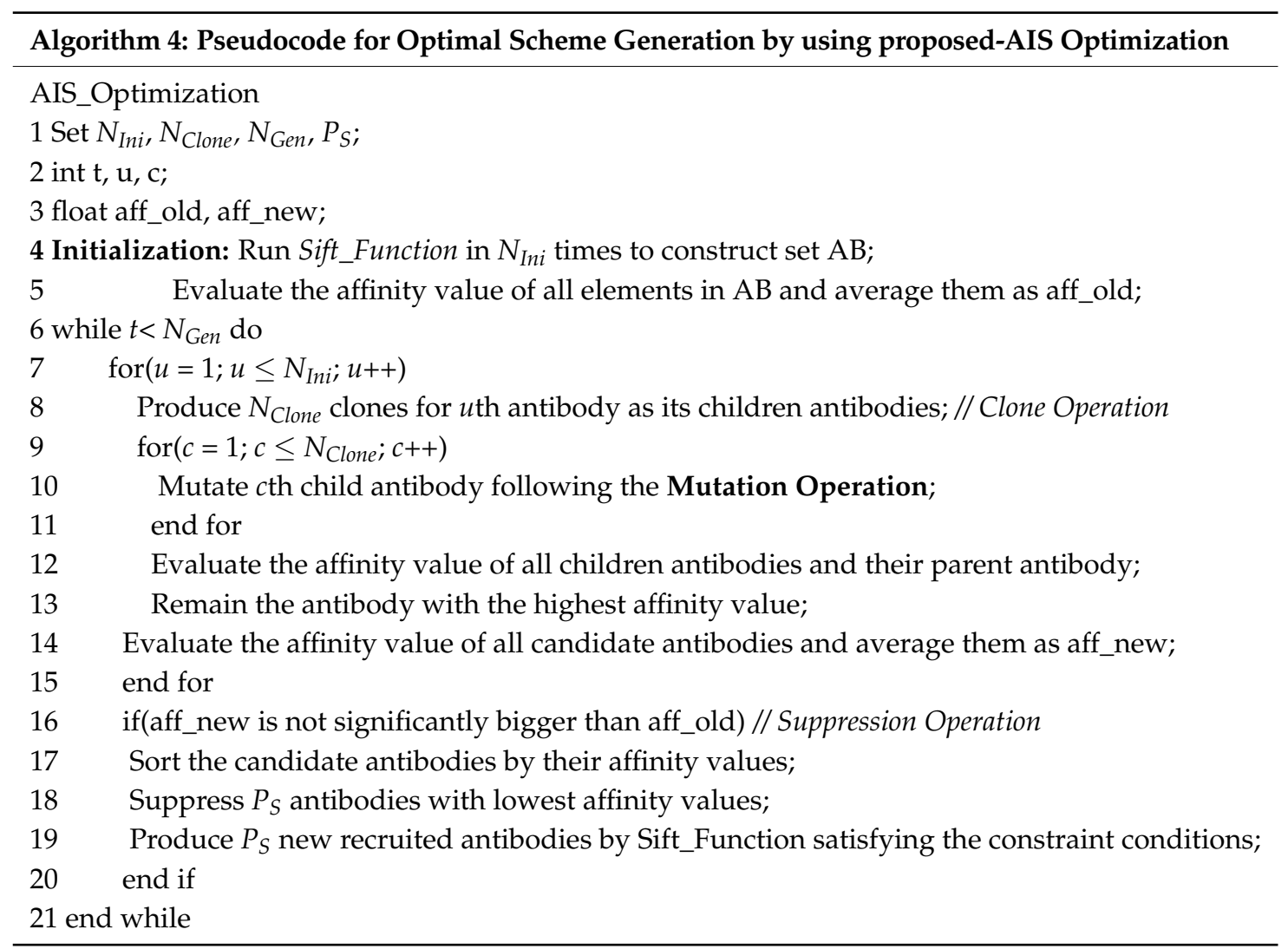




\section{Simulations and Results}

In this section, the total effective interrogation range using the proposed-AIS optimization with Sift_Function (denoted as GD-MRSOA-AIS) and without Sift_Function (denoted as MRSOA-AIS) will be simulated and compared with the state-of-the-art algorithm (LIs [14]) in two scenarios:

- $\quad$ The number of readers $N_{\text {Reader }}=15$ with fixed positions among GD-MRSOA-AIS, MRSOA-AIS and LIs. Meanwhile, the advantage of Sift_Function and proposed-AIS optimization is illustrated by comparisons of GD-MRSOA-AIS, MRSOA-AIS and LIs.

- $\quad$ Variable $N_{\text {Reader }}$ with randomized positions among GD-MRSOA-AIS, MRSOA-AIS and LIs.

During the simulations, readers are randomly distributed over an area in two dimensions with $100 \times 100 \mathrm{~m}^{2}$ (i.e., $L_{x}=100 \mathrm{~m}, L_{y}=100 \mathrm{~m}$ ). As readers communicate with tags by means of EPCGlobal C1G2, the parameter settings are same with the ones listed in Table A1 in Appendix A.

Given a certain number of readers, as the number of available time slots increases, the readers have more options to avoid simultaneously operation. In other words, for a specific number of time slots, as the number of readers increases, the total effective interrogation range will be smaller, which is caused by the heavy interferences. Without the loss of generality, $N_{\text {Slot }}$ is finally assigned as 5 in the following simulations. In addition, as the number of available frequency channels increases, the total effective interrogation range always increases since each reader has more options for selecting an available frequency channel to simultaneously operate with other readers at different frequency channels. Usually, the number of available frequency channels is set to no more than 10. Consequently, $N_{\text {Freq }}$ is assigned as 10 in the simulation. The parameters setting of proposed-AIS optimization in simulation are listed in Table 4.

Table 4. Parameter settings of proposed AIS optimization process.

\begin{tabular}{cc}
\hline Parameter & Value \\
\hline The number of available time slots $\left(N_{\text {Slot }}\right)$ & 5 \\
The number of available frequency channels $\left(N_{\text {Freq }}\right)$ & 10 \\
Initialized candidate population size $\left(N_{\text {Ini }}\right)$ & 40 \\
Cloned Multiplier $\left(N_{\text {Clone }}\right)$ & 5 \\
Maximum generation $\left(N_{\text {Gen }}\right)$ & 150 \\
Suppression percentage $\left(P_{S}\right)$ & $25 \%$ \\
\hline
\end{tabular}

\subsection{Case Study: $N_{\text {Reader }}=15, N_{\text {Slot }}=5, N_{\text {Freq }}=10$}

When $N_{\text {Reader }}=15$, the fixed positions of readers are randomized in Figure 4 , where the corresponding neighbor set for each reader is $I S_{R 1}=\{1,3\}, I S_{R 2}=\{2,5,6\}, I S_{R 3}=\{1,3\}, I S_{R 4}=\{4,7\}$, $I S_{\mathrm{R} 5}=\{5,6,8,9\}, I S_{\mathrm{R} 6}=\{2,5,6,8\}, I S_{\mathrm{R} 7}=\{4,7\}, I S_{\mathrm{R} 8}=\{5,6,8,9,10,11\}, I S_{\mathrm{R} 9}=\{5,8,9,10,11\}, I S_{\mathrm{R} 10}=$ $\{8,9,10,11\}, I S_{\mathrm{R} 11}=\{8,9,10,11\}, I S_{\mathrm{R} 12}=\{12,13\}, I S_{\mathrm{R} 13}=\{12,13\}, I S_{\mathrm{R} 14}=\{14\}$, and $I S_{\mathrm{R} 15}=\{15\}$. Here, $\mathrm{R}_{14}$ and $\mathrm{R}_{15}$ are isolated readers since $I S_{\mathrm{R} 14} \cap I S_{\mathrm{R} j}=\varnothing$ (any $j \in N R$ and $\left.j \neq 14\right)$ and $I S_{\mathrm{R} 15} \cap I S_{\mathrm{R} l}=\varnothing($ any $l \in N R$ and $l \neq 15)$. On the contrary, i.e., $I S_{\mathrm{R} 5} \cap I S_{\mathrm{R} 8} \cap I S_{\mathrm{R} 9} \cap I S_{\mathrm{R} 10} \cap I S_{\mathrm{R} 11}=\{5,8,9,10,11\}$, these readers should be scheduled to operate at different time slots or simultaneously operate but at different frequency channels.

Figure 5 shows the graphical representation of effective interrogation range for each reader with its corresponding operation time slots (where the five time slots are represented by Green, Red, Blue, Cyan and Magenta) under the operating scheduling scheme. Correspondingly, the operating scheduling scheme for each algorithm is listed in Table 5, where $\mathrm{CH}_{i}(k)=0$ means that the $i$ th reader does not operate at this $k$ th time slot. At the first time slot $\left(T_{1}\right)$ as green circles in Figure $5 a-c,\left\{R_{7}\right.$, $\left.\mathrm{R}_{9}, \mathrm{R}_{14}, \mathrm{R}_{15}\right\}$ are operating at the frequency channel of $\left\{\mathrm{CH}_{7}(1)=5, \mathrm{CH}_{9}(1)=7, \mathrm{CH}_{14}(1)=1, \mathrm{CH}_{15}(1)=\right.$ $10\}$ using GD-MRSOA-AIS as in Table 5; $\left\{R_{11}, R_{13}, R_{14}, R_{15}\right\}$ are operating at the frequency channel of $\left\{\mathrm{CH}_{11}(1)=9, \mathrm{CH}_{13}(1)=7, \mathrm{CH}_{14}(1)=1, \mathrm{CH}_{15}(1)=5\right\}$ using MRSOA-AIS; and $\left\{\mathrm{R}_{1}, \mathrm{R}_{4}, \mathrm{R}_{13}, \mathrm{R}_{15}\right\}$ are 
operating at the frequency channel of $\left\{\mathrm{CH}_{1}(1)=7, \mathrm{CH}_{4}(1)=1, \mathrm{CH}_{13}(1)=10, \mathrm{CH}_{15}(1)=3\right\}$ using LIs algorithm. Take $\left\{R_{4}, R_{7}\right\}$ as an example, $I S_{R 4} \cap I S_{R 7}=\{4,7\}$. According to Table $5, R_{4}$ is scheduled to operate at $\mathrm{TS}_{3}$ with the frequency channel of $\mathrm{CH}_{4}(3)=10$ and $\mathrm{R}_{7}$ is scheduled to operate at $\mathrm{TS}_{1}$ with the frequency channel of $\mathrm{CH}_{7}(1)=5$ by GD-MRSOA-AIS while $\mathrm{R}_{4}$ is scheduled to operate at every time slot as $\left\{\mathrm{CH}_{4}(1)=1, \mathrm{CH}_{4}(2)=3, \mathrm{CH}_{4}(3)=1, \mathrm{CH}_{4}(4)=1, \mathrm{CH}_{4}(5)=3\right\}$ and $\mathrm{R}_{7}$ is scheduled to be inactive at every time slot by LIs. Therefore in LIs, $\mathrm{R}_{4}$ is actually operating in uselessness especially for the last few time slots and it is unfair for $R_{7}$. On the contrary, GD-MRSOA-AIS schedules $R_{4}$ and $R_{7}$ corporately operating in a fair way. The same scenario is also happened in $\left\{R_{12}, R_{13}\right\}$.

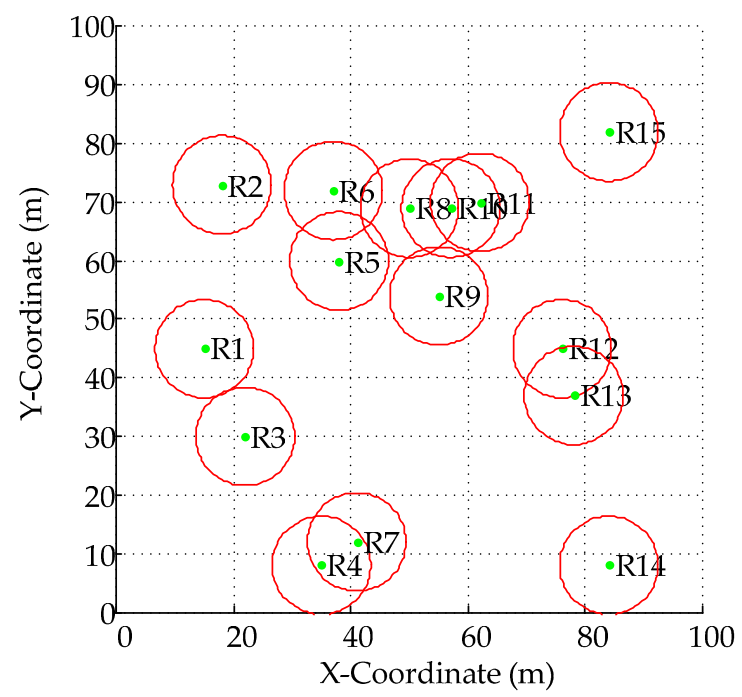

Figure 4. Fixed Location of Readers $\left(N_{\text {Reader }}=15\right)$.

Table 5. The operating schedule for 15 readers with $N_{\text {Slot }}=5$ and $N_{\text {Freq }}=10$ among three algorithms.

\begin{tabular}{|c|c|c|c|c|c|c|c|c|c|c|c|c|c|c|c|}
\hline & \multicolumn{5}{|c|}{ GD-MRSOA-AIS } & \multicolumn{5}{|c|}{ MRSOA-AIS } & \multicolumn{5}{|c|}{ LIs } \\
\hline & $\mathrm{TS}_{1}$ & $\mathrm{TS}_{2}$ & $\mathrm{TS}_{3}$ & $\mathrm{TS}_{4}$ & $\mathrm{TS}_{5}$ & $\mathrm{TS}_{1}$ & $\mathrm{TS}_{2}$ & $\mathrm{TS}_{3}$ & $\mathrm{TS}_{4}$ & $\mathrm{TS}_{5}$ & $\mathrm{TS}_{1}$ & $\mathrm{TS}_{2}$ & $\mathrm{TS}_{3}$ & $\mathrm{TS}_{4}$ & $\mathrm{TS}_{5}$ \\
\hline \multicolumn{16}{|l|}{$\mathbf{R}_{i}$} \\
\hline $\mathrm{R}_{1}$ & 0 & 0 & 0 & 0 & 0 & 0 & 6 & 7 & 1 & 1 & 7 & 0 & 0 & 3 & 5 \\
\hline $\mathrm{R}_{2}$ & 0 & 1 & 0 & 7 & 10 & 0 & 0 & 0 & 10 & 7 & 0 & 1 & 8 & 7 & 1 \\
\hline $\mathrm{R}_{3}$ & 0 & 10 & 0 & 5 & 1 & 0 & 3 & 0 & 0 & 0 & 0 & 0 & 5 & 0 & 0 \\
\hline $\mathrm{R}_{4}$ & 0 & 0 & 10 & 0 & 0 & 0 & 0 & 0 & 0 & 0 & 1 & 3 & 1 & 1 & 3 \\
\hline $\mathrm{R}_{5}$ & 0 & 0 & 1 & 0 & 0 & 0 & 0 & 0 & 0 & 0 & 0 & 0 & 0 & 0 & 1 \\
\hline $\mathrm{R}_{6}$ & 0 & 5 & 0 & 0 & 0 & 0 & 0 & 0 & 0 & 0 & 0 & 0 & 0 & 0 & 0 \\
\hline $\mathrm{R}_{7}$ & 5 & 0 & 0 & 0 & 0 & 0 & 0 & 0 & 0 & 0 & 0 & 0 & 0 & 0 & 0 \\
\hline $\mathrm{R}_{8}$ & 0 & 0 & 5 & 0 & 0 & 0 & 0 & 0 & 0 & 0 & 0 & 10 & 0 & 0 & 0 \\
\hline $\mathrm{R}_{9}$ & 7 & 0 & 0 & 0 & 0 & 0 & 2 & 10 & 5 & 0 & 0 & 0 & 0 & 0 & 8 \\
\hline $\mathrm{R}_{10}$ & 0 & 8 & 0 & 0 & 0 & 0 & 0 & 0 & 0 & 0 & 0 & 0 & 3 & 0 & 0 \\
\hline $\mathrm{R}_{11}$ & 0 & 0 & 0 & 0 & 8 & 9 & 0 & 0 & 0 & 5 & 0 & 0 & 0 & 0 & 0 \\
\hline $\mathrm{R}_{12}$ & 0 & 0 & 8 & 0 & 0 & 0 & 0 & 0 & 0 & 0 & 0 & 0 & 0 & 0 & 0 \\
\hline $\mathrm{R}_{13}$ & 0 & 3 & 0 & 0 & 0 & 7 & 0 & 0 & 3 & 0 & 10 & 7 & 7 & 10 & 7 \\
\hline $\mathrm{R}_{14}$ & 1 & 0 & 0 & 9 & 6 & 1 & 9 & 2 & 0 & 0 & 0 & 0 & 0 & 0 & 10 \\
\hline $\mathrm{R}_{15}$ & 10 & 0 & 3 & 3 & 3 & 5 & 0 & 5 & 7 & 10 & 3 & 5 & 10 & 5 & 9 \\
\hline
\end{tabular}

* In the table, $\mathrm{TS}_{k}$ stands for the $k$ th time slot from the optional values of $\left\{\mathrm{TS}_{1}, \mathrm{TS}_{2}, \mathrm{TS}_{3}, \mathrm{TS}_{4}, \mathrm{TS}_{5}\right\}$ in $N_{\text {Slot }}=5$. $\mathrm{R}_{i}$ stands for the $i$ th reader from the optional values of $\left\{\mathrm{R}_{1}, \mathrm{R}_{2}, \ldots, \mathrm{R}_{15}\right\}$ in $N_{\text {Reader }}=15$. The operating frequency channel for $i$ th reader at $k$ th time slot $\left(\mathrm{CH}_{i}(k)\right)$ is listed from the optional values of $\mathrm{CH}=\{0,1,2,3, \ldots, 10\}$ when $N_{\text {Freq }}=10$, where $C_{i}(k)=0$ means that the $i$ th reader does not work at $k$ th time slot. 


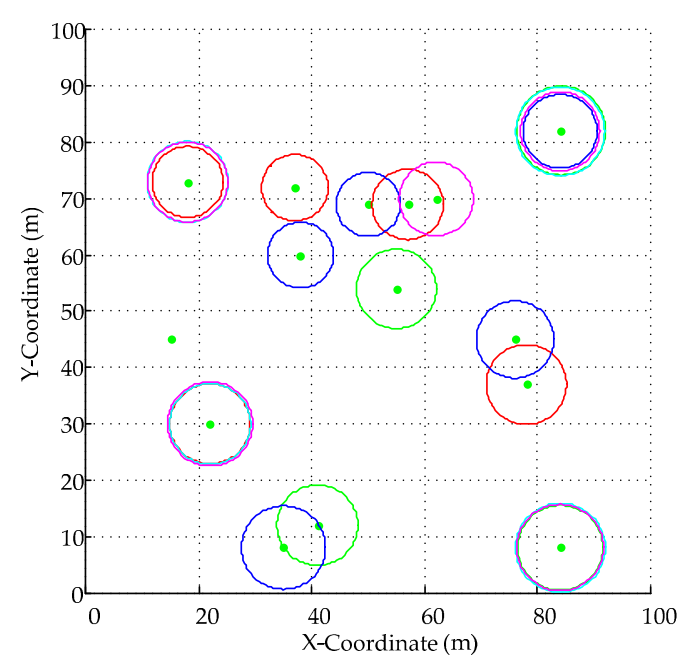

(a)

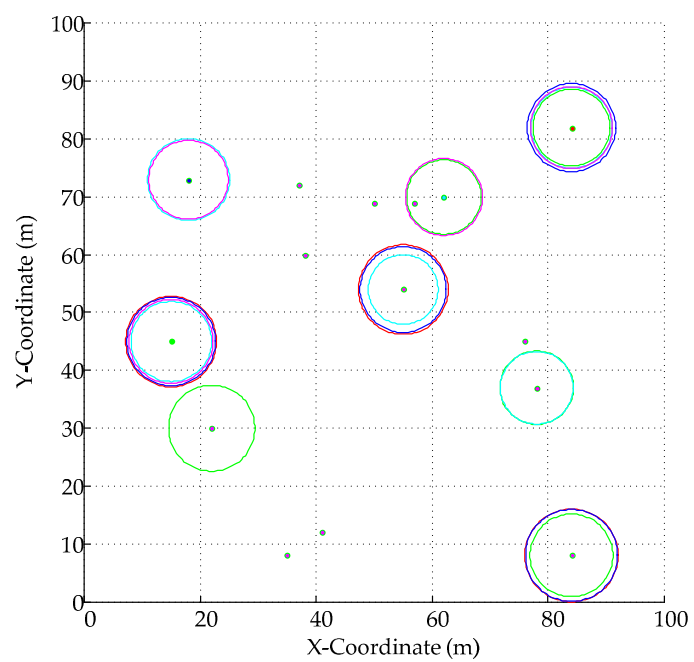

(b)

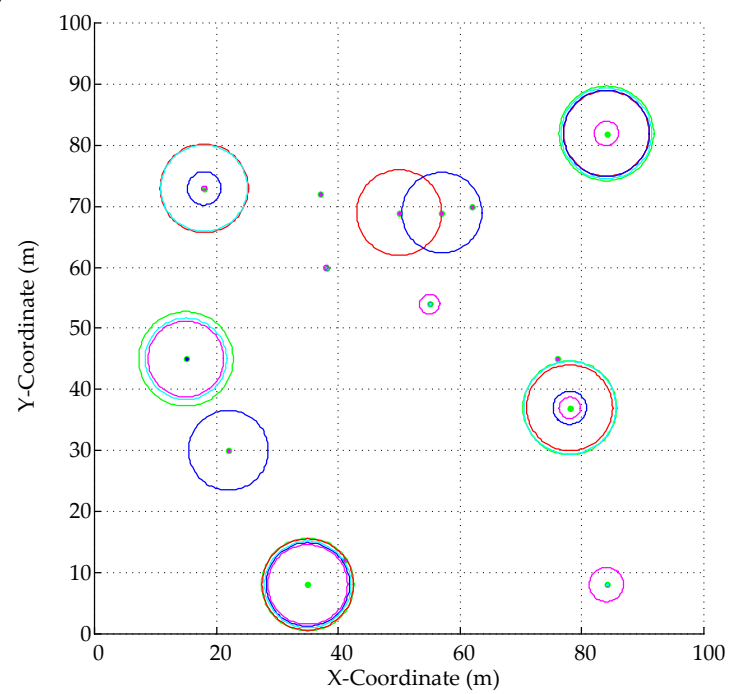

(c)

Figure 5. Graphical Representation of Optimal Operating Schedule for 15 readers with $N_{\text {Slot }}=5$ and $N_{\text {Freq }}=10$ : (a) GD-MRSOA-AIS; (b) MRSOA-AIS; and (c) LIs.

On average, the effective interrogation radius using GD-MRSOA-AIS is $2.142 \mathrm{~m}, 2.0881 \mathrm{~m}$ using MRSOA-AIS and $1.909 \mathrm{~m}$ using LIs algorithm. It seems like that the effective interrogation ranges of these three algorithms are similar. However, by introducing Sift_Function, GD-MRSOA-AIS is obviously fairer since almost all readers (except for $\mathrm{R}_{1}$ ) are scheduled to operate at different time slots and frequency channels while both of MRSOA-AIS and LIs could not. For example, $\left\{R_{4}, R_{5}, R_{6}, R_{7}, R_{8}\right.$, $\left.R_{10}, R_{12}\right\}$ are always inactive in MRSOA-AIS and $\left\{R_{6}, R_{7}, R_{11}, R_{12}\right\}$ are inactive in LIs (shown in Table 5 and Figure $5 b, c$ ), especially that $R_{6}, R_{10}, R_{11}$ are posited in crowed with more neighbors (shown in Figure 4).

To evaluate the fairness of readers during the operating scheduling, a general purpose quantitative measure of fairness called Jain Index [26] is imported as Equation (11), where $N_{\text {Reader }}$ denotes the number of readers, $\omega_{i}$ is the $0-1$ function to indicate whether the reader $\mathrm{R}_{i}$ operates at least once at these $N_{\text {Slot }}$ time slots or not, and $N_{\text {Neighbor }}(i)$ is the cardinality of $I S_{R i}$. The $I_{\text {Jain }}$ ranges from 0 to 1 : the higher $I_{\text {Jain }}$ means that the corresponding optimal scheduling scheme is fairer. Obviously, $\mathrm{R}_{i}$ with larger $N_{\text {Neighbor }}(i)$ will make more contribution to obtain a bigger $I_{\text {Jain }}$. Therefore, the Jain Index could express the fairness of an optimal scheduling scheme. 


$$
I_{\text {Jain }}=\frac{\sum_{i=1}^{N_{\text {Reader }}} \omega_{i} \times N_{\text {Neighbor }}(i)}{N_{\text {Reader }} \times N_{\text {Reader }}}
$$

As a result, GD-MRSOA-AIS becomes the fairest one with highest Jain Index of $I_{\text {Jain }}=18.22 \%$, while the $I_{\text {Iain }}$ of MRSOA-AIS is $8.89 \%$ and the $I_{\text {Iain }}$ of LIs is $14.67 \%$ in the same scenario.

In Figure 6, the initial average affinity value of GD-MRSOA-AIS starts from 450, which is much higher than that of the other two from 200. This obvious difference is owing to the contending mechanism of Sift_Function for time slots selection. Additionally, the average affinity value of both GD-MRSOA-AIS and MRSOA-AIS are much higher than LIs, where MRSOA-AIS rockets up to a high affinity value and then it stabilizes in the similar average affinity value like GD-MRSOA-AIS, which dues to the multiple mutation operations in the proposed-AIS. To achieve the average affinity value of 600, with GD-MRSOA-AIS and MRSOA-AIS, it costs around 50 generations while with LIs it is more than 150 generations. Therefore, the proposed-AIS also reduces the time consumption of optimization process.

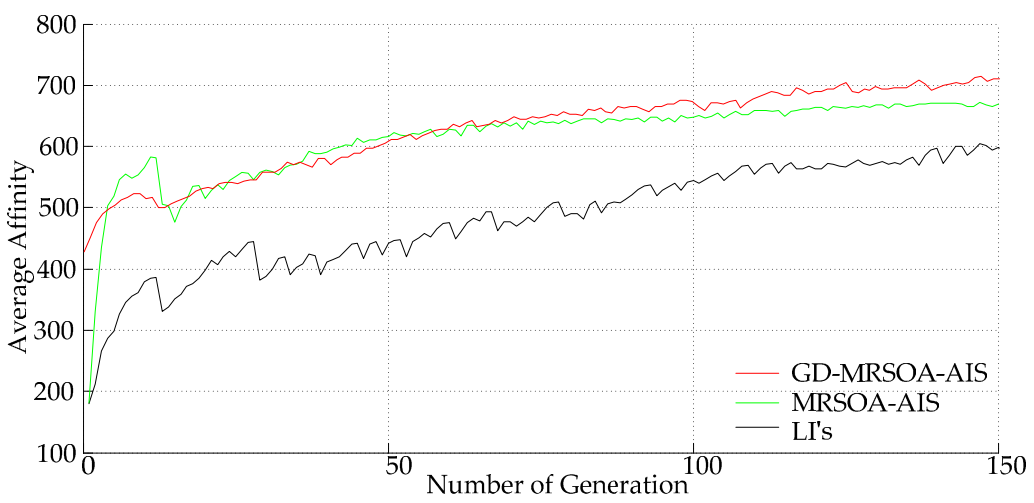

Figure 6. Affinity value versus number of generations.

\subsection{Variable $N_{\text {Reader }}$ with Fixed $N_{\text {Slot }}=5$ and $N_{\text {Freq }}=10$ in Randomized Positions}

For a given $N_{\text {Reader }}$ readers with $N_{\text {Slot }}=5$ and $N_{\text {Freq }}=10$ in the area of $100 \times 100 \mathrm{~m}^{2}$, the position of each reader is randomly located. To ensure the convergence of the results, all the simulations are repeated for 50 times with 50 randomly positions. For an easier understanding, the results are listed in five indices (the best, the worst, and the mean of total effective interrogation area, the average radius and Jain Index) among GD-MRSOA-AIS, MRSOA-AIS and LIs algorithm, which are represented in Table 6.

When $N_{\text {Reader }}=4$ with $N_{\text {Slot }}=5$ available time slots and $N_{\text {Freq }}=10$ frequency channels, readers are easily scheduled to operate at different time slots and frequency channels. Additionally, they could be deployed so sparsely that they are hardly interfered each other in such a large zone $\left(100 \times 100 \mathrm{~m}^{2}\right)$. Therefore, the Jain Index is failed to measure the fairness. However, limited by the self-rated-power, the interrogation range is also finite. As a result, the total effective interrogation range is not large enough. As the number of readers increases, such as $N_{\text {Reader }}=24$ and $N_{\text {Reader }}=28$, positions of readers become much denser, the readers could not make full use of the frequency channels and the time slots, and then the individual effective interrogation range is declining and the total effective interrogation range relies on the cooperation among readers operating.

From Table 6, we can see that the Jain Index is fluctuated up and down. For the former situations (i.e., $N_{\text {Reader }}=8, N_{\text {Reader }}=12, N_{\text {Reader }}=16$ ), each reader has fewer neighbors since they are sparsely distributed, which declines the Jain Index value even if all of the readers are scheduled to operate at least once. For the latter situations (i.e., $N_{\text {Reader }}=24, N_{\text {Reader }}=28$ ), readers are located in denser positions and so each reader has more neighbors, but some readers might be not scheduled to operate even once 
$\left(\omega_{i}=0\right)$ which also decreases the Jain Index value. Even so, the Jain Index value of GD-MRSOA-AIS is also higher than MRSOA-AIS and LIs with the same number of readers.

Table 6. The comparison results of the total effective interrogation range with $N_{\text {Slot }}=5$ and $N_{\text {Freq }}=10$.

\begin{tabular}{|c|c|c|c|c|c|c|}
\hline$N_{\text {Reader }}$ & Algorithm & $\begin{array}{c}\text { Best } \\
\left(\text { Unit: } \mathbf{m}^{2}\right) \\
\end{array}$ & $\begin{array}{c}\begin{array}{c}\text { Worst } \\
\left(\text { Unit: } \mathbf{m}^{2}\right)\end{array} \\
\end{array}$ & $\begin{array}{c}\text { Mean } \\
\left(\text { Unit: } \mathbf{m}^{2}\right) \\
\end{array}$ & $\begin{array}{c}\text { Average Radius } \\
\text { (Unit: m) }\end{array}$ & $\begin{array}{c}\text { Jain Index } \\
\left(I_{\text {Jain }}\right)\end{array}$ \\
\hline \multirow{3}{*}{4} & GD-MRSOA-AIS & 3886.166 & $2459.977^{*}$ & $3190.954 *$ & $6.906 *$ & - \\
\hline & MRSOA-AIS & $3976.631 *$ & 1918.756 & 3078.580 & 6.784 & - \\
\hline & LIs & 3570.661 & 1512.397 & 3041.527 & 6.573 & - \\
\hline \multirow{3}{*}{8} & GD-MRSOA-AIS & $4392.362 *$ & $3287.613 *$ & $3907.504 *$ & $4.308 *$ & $42.19 \%$ * \\
\hline & MRSOA-AIS & 4293.650 & 3180.301 & 3790.565 & 4.174 & $25.00 \%$ \\
\hline & LIs & 4316.239 & 3263.520 & 3859.630 & 4.252 & $29.69 \%$ \\
\hline \multirow{3}{*}{12} & GD-MRSOA-AIS & $3910.000 *$ & $3328.207^{*}$ & $3539.014 *$ & $2.670 *$ & $27.08 \%$ * \\
\hline & MRSOA-AIS & 3765.571 & 3014.043 & 3481.625 & 2.620 & $13.89 \%$ \\
\hline & LIs & 3671.977 & 2977.352 & 3428.592 & 2.639 & $18.06 \%$ \\
\hline \multirow{3}{*}{16} & GD-MRSOA-AIS & 3780.408 * & 3356.667 * & $3533.103 *$ & $2.027 *$ & $27.34 \%$ * \\
\hline & MRSOA-AIS & 3612.826 & 2901.954 & 3471.431 & 1.974 & $19.92 \%$ \\
\hline & LIs & 3437.009 & 3128.828 & 3270.978 & 1.963 & $21.88 \%$ \\
\hline \multirow{3}{*}{20} & GD-MRSOA-AIS & 3331.845 & 2935.414 * & 3133.686 & 1.443 & $70.50 \%$ * \\
\hline & MRSOA-AIS & $3369.615 *$ & 2901.954 & $3160.941 *$ & $1.463 *$ & $40.25 \%$ \\
\hline & LIs & 2869.225 & 2297.590 & 2528.864 & 1.346 & $45.50 \%$ \\
\hline \multirow{3}{*}{24} & GD-MRSOA-AIS & 3232.966 * & $2550.474 *$ & $2909.812 *$ & $1.229 *$ & $68.06 \% *$ \\
\hline & MRSOA-AIS & 3206.098 & 2279.184 & 2830.733 & 1.195 & $43.75 \%$ \\
\hline & LIs & 2151.093 & 1874.913 & 2035.651 & 1.020 & $42.36 \%$ \\
\hline \multirow{3}{*}{28} & GD-MRSOA-AIS & $3068.395 *$ & 2436.688 & $2737.177^{*}$ & 0.999 * & $64.29 \%$ * \\
\hline & MRSOA-AIS & 2675.789 & $2586.912 *$ & 2616.538 & 0.923 & $35.71 \%$ \\
\hline & LIs & 1859.409 & 1532.377 & 1670.742 & 0.814 & $32.14 \%$ \\
\hline
\end{tabular}

* The best one for each comparison.

In addition, Table 6 shows that the best, the worst and the mean of total effective interrogation range between GD-MRSOA-AIS and MRSOA-AIS are similar but larger than the ones of LIs; meanwhile, the average radius of effective interrogation range for each operating readers with GD-MRSOA-AIS and MRSOA-AIS is also larger than that with LIs. The total effective interrogation range is declined fast by using LIs when the number of readers increases while it is much slower by using both GD-MRSOA-AIS and MRSOA-AIS. For example, when $N_{\text {Reader }}=20$, the mean effective interrogation range with GD-MRSOA-AIS is $3133.686 \mathrm{~m}^{2}$, the best one as $3331.845 \mathrm{~m}^{2}$ and the worst one as $2935.414 \mathrm{~m}^{2}$; the mean value with LIs is only $2528.864 \mathrm{~m}^{2}$, the best one as $2869.225 \mathrm{~m}^{2}$ and the worst one as $2297.590 \mathrm{~m}^{2}$. When $N_{\text {Reader }}=28$, with GD-MRSOA-AIS, the mean value is glided down to $2737.177 \mathrm{~m}^{2}$, the best one as $3068.395 \mathrm{~m}^{2}$ and the worst one as $2436.688 \mathrm{~m}^{2}$; with LIs, the mean value is slumped down as $1670.742 \mathrm{~m}^{2}$, the best one as $1859.409 \mathrm{~m}^{2}$ and the worst one as $1532.377 \mathrm{~m}^{2}$.

In conclusion, GD-MRSOA-AIS could schedule multiple readers operating in fairness with lower multiple-reader interfering and large effective interrogation range, where the fairness with lower multiple-reader interfering dues to the geometric distribution of Sift_Function and the large effective interrogation range dues to the proposed-AIS optimization process. As a result, the deployment of eight readers with five available time slots and 10 available frequency channels is the most economical way to maximize the total effective interrogation range in $100 \times 100 \mathrm{~m}^{2}$, especially using GD-MRSOA-AIS.

\section{Conclusions}

This paper refers to the multiple-reader interference problem in the multiple-reader environment (MRE) and formulates a resource allocation model with the consideration of interference avoidance in SINR under EPCGlobal C1G2 standard, where the allocable resources include available time slots and frequency channels. Based on this resource allocation model, GD-MRSOA-AIS is further 
proposed. In GD-MRSOA-AIS, geometric distribution replaces the uniform distribution to produce the feasible scheduling schemes, where the reader, which is interfered by large quantity of readers, has more opportunities to be allocated in an operating time slot. To optimize these feasible scheduling schemes, the artificial immune system optimization including immune clone, immune mutation and immune suppression is further introduced. During the mutation operation for antibodies, there are two optional mutation operators depending on the affinity values. According to the simulation results, GD-MRSOA-AIS could obtain an optimal and fairer readers collaboratively operating scheduling scheme, and it could output a larger total effective interrogation range with higher convergence than the state-of-the-art one.

Although GD-MRSOA-AIS indeed enhances the effective interrogation range with lower interferences by fairer operating scheme, it still has shortcomings:

- It only focuses on the scenario of multiple readers in known position with randomized tags in fixed positions, and ignores the new entrancing tags with variable population.

- It only solves the resource allocation problem for multiple readers scheduling to interrogate the tags in centralized MRE, but ignores the identification problem (i.e., tag-to-tag collision) in data transmission process.

Therefore, the next work will reconstruct a resource allocation model by considering the variable population of tags and identification problem with an enhanced scheduling-based algorithm.

Acknowledgments: This article is supported by National Natural Science Foundations of China (Nos. 61303207, 61503273 and 61472271).

Author Contributions: Litian Duan and Fu Duan conceived and designed the experiments; Fu Duan performed the simulations; Litian Duan, Fu Duan and Zizhong John Wang analyzed the data; and Litian Duan and Zizhong John Wang wrote the paper.

Conflicts of Interest: The authors declare no conflict of interest.

\section{Appendix A}

Assume that readers with the rated power of $30 \mathrm{~dB} \mathrm{~m}(1 \mathrm{~W})$ are operating at the frequency of $902-928 \mathrm{MHz}$ with the bandwidth of $500 \mathrm{KHz}$ and noise power of $-90 \mathrm{~dB} \mathrm{~m}$ according to EPCGlobal C1G2 [22]. The corresponding parameters are listed in Table A1.

Table A1. Parameter value under EPCGlobal C1G2 standard.

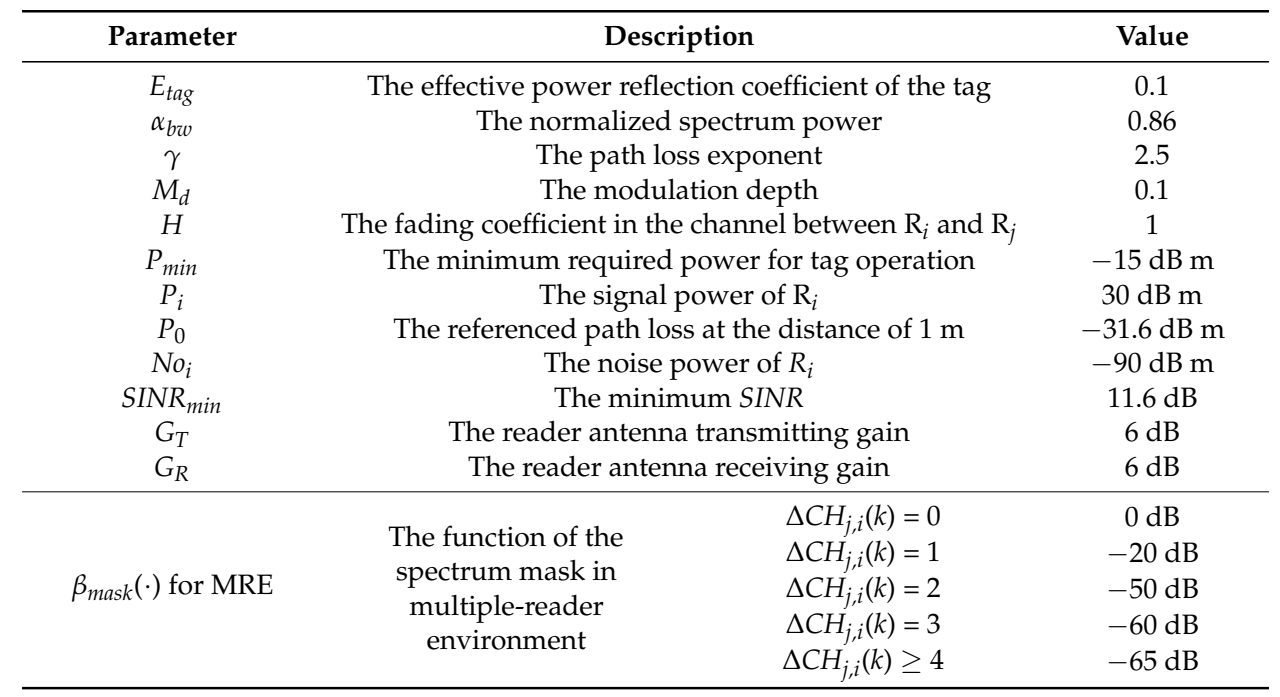

For any $\mathrm{R}_{i}$ and $\mathrm{R}_{j}$, the effective interrogation range might be overlapped if the distance between these two readers is close enough. For this scenario, readers are not preferred to operate simultaneously 
even though they are at different frequency channels. To determine this boundary distance, it is derived as follows.

$$
\begin{aligned}
& \text { According to Equation (7), } \\
& r_{i}=\left(\frac{B P}{I_{j} \times \beta_{\text {mask }}\left(\Delta C H_{j, i}(k)\right) \times d_{j, i}^{-\gamma}+S N_{i}}\right)^{\frac{1}{2 \gamma}}, \\
& \text { where } B P=\alpha_{b w} E_{\text {tag }} P_{i} G_{T} G_{R} P_{0}{ }^{2}, I_{j}=S I N R_{\min } h P_{j} G_{T} G_{R} P_{0}, S N_{i}=S I N R_{\min } \times N o_{i} \text {, } \\
& \text { Suppose that } r_{i} \leq \frac{d_{j, i}}{2} \text {, } \\
& \Rightarrow \\
& \left(\frac{B P}{I_{j} \times \beta_{\text {mask }}\left(\Delta C H_{j, i}(k)\right) \times d_{j, i}^{-\gamma}+S N_{i}}\right)^{\frac{1}{2 \gamma}} \leq \frac{d_{j, i}}{2}, \\
& \Rightarrow \\
& \text { Suppose that } A=I_{j} \times \beta_{\text {mask }}\left(\Delta C H_{j, i}(k)\right), B=S N_{i} \text {, } \\
& \text { then }\left(\frac{B P}{A \times d_{j, i}^{-\gamma}+B}\right)^{\frac{1}{2 \gamma}} \leq \frac{d_{j, i}}{2} \text {, } \\
& \Rightarrow \\
& \text { Suppose that } f\left(d_{j, i}\right)=A \times d_{j, i}^{\gamma}+B \times d_{j, i}^{2 \gamma}-4^{\gamma} \times B P \text {, } \\
& \text { and let } x=d_{j, i}^{\gamma} \text {, } \\
& \text { then } f(x)=A x+B x^{2}-4^{\gamma} B P \text {, } \\
& \Rightarrow \\
& \Delta=A^{2}+4 \times B \times 4^{\gamma} B P>0, \\
& x=\frac{-A \pm \sqrt{\Delta}}{2 B}, \\
& \text { let } x \geq 0 \\
& \Rightarrow \\
& d_{j, i}=\sqrt[\gamma]{x} \\
& \Rightarrow
\end{aligned}
$$

\section{References}

1. Vlachos, I.P. A Hierarchical Model of the Impact of RFID Practices on Retail Supply Chain Performance. Expert Syst. Appl. 2014, 41, 5-15. [CrossRef]

2. Arkan, I.; Landeghem, H.V. Evaluating the Performance of a Discrete Manufacturing Process using RFID: A Case Study. Robot. Comput. Integr. Manuf. 2013, 29, 502-1512. [CrossRef]

3. Enrique, V.; Antonio, A.; Carlos, C. Evolution of RFID Applications in Construction: A Literature Review. Sensors 2015, 15, 15988-16008.

4. Decarli, N.; Guidi, F.; Dardari, D. Passive UWB RFID for Tag Localization: Architectures and Design. IEEE Sens. J. 2016, 16, 1385-1397. [CrossRef]

5. Bueno-Delgado, M.V.; Pavon-Marino, P. A Centralized and Aligned Scheduler for Passive RFID Dense Reader Environments working under EPCglobal Standard. Simul. Model. Pract. Theory 2013, 34, 172-185. [CrossRef]

6. Wang, D.; Wang, J.; Zhao, J.Y. A Novel Solution to the Reader Collision Problem in RFID System. In Proceedings of the IEEE International Conference Wireless Communication, Networking Mobile Computing, Wuhan, China, 22-24 September 2006; pp. 1-4. 
7. Birari, S.M.; Lyer, S. Pulse: A MAC Protocol for RFID Networks. In Proceedings of the International Conference on Embedded and Ubiquitous Computing (EUC 2005), Nagasaki, Japan, 6-9 December 2005; pp. 1036-1046.

8. Eom, J.B.; Yim, S.B.; Lee, T.J. An Efficient Reader Anti-collision Algorithm in Dense RFID Networks with Mobile RFID Readers. IEEE Trans. Ind. Electron. 2009, 56, 2326-2336.

9. Song, I.C.; Hong, S.H.; Chang, K.H. An Improved Reader Anti-collision Algorithm based on Pulse Protocol with Slot Occupied Probability in Dense Reader Mode. In Proceedings of the IEEE Vehicular Technology Conference, Barcelona, Spain, 26-29 April 2009; pp. 1-5.

10. Waldrop, J.; Engels, D.W.; Sarma, S.E. Colorwave: A MAC for RFID Reader Networks. In Proceedings of IEEE International Conference on Wireless Communications and Networking (WCNC), New Orleans, LA, USA, 20-20 March 2003; pp. 1701-1704.

11. Ho, J.; Engels, D.W.; Sarma, S.E. HiQ: A Hierarchical Q-learning Algorithm to Solve the Reader Collision Problem. In Proceedings of the International Symposium on Applications and the Internet Workshops (SAINT), Phoenix, AZ, USA, 23-27 January 2006; pp. 1-4.

12. Seo, H.; Lee, C. A New GA-based Resource Allocation Scheme for a Reader-to-reader Interference Problem in RFID System. In Proceedings of the IEEE International Conference on Communications (ICC), Cape Town, South Africa, 23-27 May 2010; pp. 1-5.

13. Chen, H.N. RFID Network Planning using a Multi-swarm Optimizer. J. Netw. Comput. Appl. 2011, 34, 888-901. [CrossRef]

14. Li, Z.H.; He, C.H. Optimal Scheduling-based RFID Reader-to-reader Collision Avoidance Method using Artificial Immune System. Appl. Soft Comput. 2013, 13, 2557-2568. [CrossRef]

15. Li, Z.H.; Li, J.M.; He, C.H. Artificial Immune Network-based Anti-collision Algorithm for Dense RFID Readers. Expert Syst. Appl. 2014, 41, 4798-4810. [CrossRef]

16. Li, Z.H.; Li, J.M.; He, C.H.; Tang, C.P.; Zhou, J.Y. RFID Reader-to-reader Collision Avoidance Model with Multiple-density Tag Distribution solved by Artificial Immune Network Optimization. Appl. Soft Comput. 2013, 30, 249-264. [CrossRef]

17. Li, Z.H.; He, C.H.; Huang, X. RFID Reader Anti-collision Algorithm using Adaptive Hierarchical Artificial Immune System. Expert Syst. Appl. 2014, 41, 2126-2133. [CrossRef]

18. Tang, S.J.; Yuan, J.; Li, X.Y.; Chen, G.H.; Liu, Y.H.; Zhao, J.Z. Raspberry: A Stable Reader Activation Scheduling Protocol in Multi-reader RFID Systems. In Proceedings of the 17th IEEE International Conference on Network Protocols (ICNP), Princeton, NJ, USA, 13-16 October 2009; pp. 304-313.

19. Kim, D.Y.; Yoon, H.G.; Jang, B.J.; Yook, J.G. Effects of Reader-to-reader Interference on the UHF RFID Interrogation Range. IEEE Trans. Ind. Electron. 2009, 56, 2337-2346.

20. Vita, D.; Iannaccone, G. Design Criteria for the RF Section of UHF and Microwave Passive RFID Transponders. IEEE Trans. Microw. Theory Tech. 2005, 53, 2978-2990. [CrossRef]

21. Cha, K.; Ramachandran, A.; Jagannathan, S. Adaptive and Probabilistic Power Control Algorithms for Dense RFID Reader Network. In Proceedings of the IEEE on Networking, Sensing and Control (ICNSC), Ft. Lauderdale, FL, USA, 23-25 April 2006; pp. 474-479.

22. EPC Global. UHF Air Interface Protocol Standard Generation2/Version2. Available online: http://www.gs1. org/gsmp/kc/epcglobal/uhfc1g2 (accessed on 14 November 2016).

23. Safa, H.; I-Hajj, W.E.; Meguerditchian, C. A Distributed Multi-channel Reader Anti-collision Algorithm for RFID Environments. Comput. Commun. 2015, 64, 44-56. [CrossRef]

24. Tay, Y.C.; Jamieson, K.; Balakrishnan, H. Collision-Minimizing CSMA and its Applications to Wireless Sensor Networks. IEEE J. Sel. Areas Commun. 2004, 22, 1048-1057. [CrossRef]

25. Bueno-Delgado, M.V.; Ferrero, R.; Gandino, F.; Pavon-Marino, P.; Rebaudengo, M. A Geometric Distribution Reader Anti-Collision Protocol for RFID Dense Reader Environments. IEEE Trans. Autom. Sci. Eng. 2013, 10, 296-306. [CrossRef]

26. Ferrero, R.; Gandino, F.; Montrucchio, B.; Rebaudengo, M. A Fair and High Throughput Reader-to-reader Anti-collision Protocol in Dense RFID Networks. IEEE Trans. Ind. Inf. 2012, 8, 697-706. [CrossRef]

(C) 2016 by the authors; licensee MDPI, Basel, Switzerland. This article is an open access article distributed under the terms and conditions of the Creative Commons Attribution (CC-BY) license (http://creativecommons.org/licenses/by/4.0/). 